\title{
A VARIATIONAL ITERATION METHOD FOR SOLVING ORDINARY DIFFERENTIAL EQUATIONS USING THE ABOODH TRANSFORM
}

\section{MOHAMED ELARBI BENATTIA AND KACEM BELGHABA}

(Received 21 January 2019; Revision Accepted 6 March 2019)

\begin{abstract}
In this article we propose a modification of the Variational Iteration Method (VIM) by adopting Aboudh transformation method. With this method a Lagrange multiplier is determined and the technique enhances the anatical solution of both the linear and nonlinear ordinary differential equations. Adoption of the Adomian series is also demonstrated. Some examples are given to illustrate the process.
\end{abstract}

KEYWORDS: Aboodh Transform, Ordinary differential Equation, Partial Derivative, Approximate Solution.

\section{INTRODUCTION}

The variational iteration method (VIM) is an analytical technique that has been widely used in the last ten years [3, 11, 12], and the results in the approximate solutions have a high accuracy. Many researchers are making efforts for its developments $[5,6,10]$. In this article, the transformation of Aboodh is adopted in some know analytical methods, the combination of Aboudh and the homotopy perturbation method is used to simplify the solution process and improve the accuracy of the solution. We give a new modified variational iteration method, based on the theory of variational iteration and Aboudh transform. In this work, we will use this method to solve some ordinary differential equations, including non-linear ones and differential equations with variable coefficients. The transformation of Aboodh and the non-linear term will be treated by the Adomian series. The results show that this new modification scheme.

\section{THE ABOODH TRANSFORM}

The Aboodh transform is a new integral transform similar to the Laplace transform and other integral transforms that are defined in the time domain $t \geq 0$, such as the Sumudu transform, the Natural transform and the Elzaki transform, respectively.

An Aboodh transform is defined for functions of exponential order. We consider functions in the set $F$ defined by :

$$
F=\left\{\begin{array}{l}
f(t),|f(t)|<M e^{-v t}, t \in[0,+\infty[ \\
, M, k_{1}, k_{2}>0, k_{1} \leq v \leq k_{2}
\end{array}\right\}
$$

For a given function in the set $F, M$ must be finite number and $k_{1}, k_{2}$, may be finite or infinite with variable $v$ defined as $k_{1} \leq v \leq k_{2}$. Then, the Aboodh transform denoted by the operator $A(:)$ is defined by the integral equation:

$$
A[f(t)]=\frac{1}{v} \int_{0}^{+\infty} f(t) e^{-v t} d t, . t \geq 0
$$

Mohamed Elarbi Benattia, Laboratory of Mathematics and its Applications (LAMAP), University of Oran 1, P.O. Box 1524, Oran 31000, Algeria.

Kacem Belghaba, Laboratory of Mathematics and its Applications (LAMAP), University of Oran 1, P.O. Box 1524, Oran 31000, Algeria. 
Standard Aboodh transform for some special functions found are given below in Table 1

\begin{tabular}{|l|l|}
\hline$f(t)$ & $A[f(t)]$ \\
\hline $\mathbf{1}$ & $\frac{1}{v^{2}}$ \\
\hline$t$ & $\frac{1}{v^{3}}$ \\
\hline$t^{n}, n \geq 1$ & $\frac{n !}{v^{n+2}}$ \\
\hline$e^{a t}$ & $\frac{1}{v^{2}-a v}$ \\
\hline $\sin (a t)$ & $\frac{1}{v\left(v^{2}+a^{2}\right)}$ \\
\hline $\cos (a t)$ & $\frac{1}{\left(v^{2}+a^{2}\right)}$ \\
\hline $\sinh (a t)$ & $\frac{1}{v\left(v^{2}-a^{2}\right)}$ \\
\hline$t \cosh (a t)$ & $\frac{1}{\left(v^{2}-a^{2}\right)}$ \\
\hline
\end{tabular}

TABLE1: Aboodh transform of some functions.

Theorem1: Aboodh transform of some partial derivatives:

(i) $A\left[f^{\prime}(t)\right]=v A[f(t)]-\frac{f(0)}{v}$

(ii) $A\left[f^{\prime \prime}(t)\right]=v^{2} A[f(t)]-\frac{f^{\prime}(0)}{v}-f(0)$

(iii) $A\left[f^{(n)}(t)\right]=v^{n} A[f(t)]-\sum_{k=0}^{n-1} \frac{f^{(k)}(0)}{v^{2-n+k}}$.

Remark1: The Aboodh transform is linear, i.e., if $\alpha$ and $\beta$ are any constants, $f(t)$ and $g(t)$ are functions defined over the set $F$ above, then

$A[\alpha f(t)]+A[\beta g(t)]=\alpha A[f(t)]+\beta A[g(t)]$

2 Aboodh Transform Method:

Let us consider the general nonlinear ordinary differential equation (ODE) of the form

$\frac{d^{m} w(t)}{d t^{m}}+P[w(t)]+Q[w(t)]=g(t), m=1,2, \ldots$

with initial condition

$w^{k}(0)=\left.\frac{d^{k} w(t)}{d t^{k}}\right|_{t=0}=a_{k}, k=0 ; 1, \ldots .(m-1) \ldots$

where $\frac{d^{m} w(t)}{d t^{m}}$ is the derivative of $w(t)$ of order $m$ which is invertible, $P$ is the nonlinear term, $Q$ is a linear operator and $g(t)$ a known continuous function. 
Inspired by Wu's[3] method, if we take Aboodh transform on both sides of Eqs (4) and (5), the linear part with constant coefficients is then transferred into an algebraic one, so that we can identify the Lagrange multiplier in a more straightforward way. Now, we extend this idea to find the unknown Lagrange multiplier. Appling the Aboodh's transform to eq (4), and using the results of eq (3), we have eqn (6):

$A\left[\frac{d^{n} w(t)}{d t^{n}}\right]+A[P w(t)+Q w(t)]=A[g(t)]$

But

$A\left[\frac{d^{m} w(t)}{d t^{m}}\right]=v^{m} W(t)-\sum_{k=0}^{m-1} \frac{w^{(k)}(0)}{v^{2-m+k}}$

So, we have

$v^{m} W(v)-\sum_{k=0}^{m-1} \frac{w^{(k)}(0)}{v^{2-m+n}}-A[g(t)]+A[P w(t)+Q w(t)]=0 \ldots$

be used to suggest the main iterative scheme involving the Lagrange multiplier as

$W_{n+1}(v)=W_{n}(v)+\lambda(v)\left[v^{m} W(v)\right]-\lambda(v) \sum_{k=0}^{m-1} \frac{w^{(k)}\left(0^{+}\right)}{v^{2-m+k}}$

$+\lambda(v)[A[g(t)]+A[P w(t)+Q w(t)]]$

taking the classical variation operator on both sides of eq(9)

$\delta W_{n+1}(v)=\delta W_{n}(v)+\lambda(v)\left[v^{m} W_{n}(v)\right]$

one can derive a Lagrange multiplier

$\lambda=-\frac{1}{v^{m}} \ldots \ldots . . .(11)$

With eq(11) and the inverse Aboodh transform $A^{-1}$ the iteration formula eq(9) can be explicitly given as $w_{n+1}(t)=w_{n}(t)+A^{-1}\left[-\frac{1}{v^{m}}\left[v^{m} W(v)-\sum_{k=0}^{m-1} \frac{w^{(k)}\left(0^{+}\right)}{v^{2-m+k}}\right]\right]$

$+A^{-1}\left[\frac{1}{v^{m}}([A[g(t)]+A[P w(t)]+A[Q w(t)]])\right]$

$=w_{0}(t)+A^{-1}\left[-\frac{1}{v^{m}}[A[P w(t)]+A[Q w(t)]]\right.$

where $\mathrm{w}_{0}(t)$ is an initial approximation of $\mathrm{Eq}(4)$, and

$$
\begin{aligned}
& w_{0}(t)=A^{-1}\left[\sum_{k=0}^{m-1} \frac{w^{(k)}\left(0^{+}\right)}{v^{2+k}}+\frac{1}{v^{m}} A[g(t)]\right] \\
& =w_{0}+w_{0}^{\prime} t+\ldots . .+\frac{w^{m-1}(x, 0) t^{m-1}}{(m-1) !}+A^{-1}\left[\frac{1}{v^{m}} A[g(t)]\right]
\end{aligned}
$$

\section{Illustrative Examples}

In this section, we apply the above defined method to solve both ordinary differential equations .

\subsection{Linear and Nonlinear ODEs:}

Exemple1: We consider the following simple linear first order differential equation

$$
\frac{d w(t)}{d t}=a w(t), . . w(0)=w_{0}
$$

which has the exact solution $w(t)=w_{0} e^{a t}$. Applying the procedure in sub section (2.2) we get the successive formula

$$
\left\{\begin{array}{l}
w(0)=w_{0} \\
w_{n+1}(t)=A^{-1}\left[w_{0}+\frac{1}{v} A\left[a w_{n}(t)\right]\right]
\end{array}\right.
$$


and the approximate solution

$$
\left\{\begin{array}{l}
w_{0}(t)=A^{-1}\left[\frac{1}{v^{2}} w_{0}\right]=w_{0} . \\
w_{1}(t)=w_{0}+A^{-1}\left[\frac{1}{v} A\left[a w_{0}(t)\right]\right]=w_{0}+A^{-1}\left[\frac{1}{v} A\left[a w_{0}\right]\right] \\
=w_{0}+A^{-1}\left[\frac{1}{v}\left[a w_{0} \frac{1}{v^{2}}\right]\right]=w_{0}+a w_{0} A^{-1}\left[\frac{1}{v^{3}}\right]=w_{0}+a w_{0} t . \\
w_{2}(t)=w_{0}+A^{-1}\left[\frac{1}{v} A\left[a w_{1}(t)\right]\right] \\
=w_{0}+A^{-1}\left[\frac{1}{v} A\left[a w_{0}+a^{2} w_{0} t\right]\right] \\
=w_{0}+A^{-1}\left[\frac{1}{v} A\left[a w_{0}\right]+\frac{1}{v} A\left[a^{2} w_{0} t\right]\right] \\
=w_{0}+a w_{0} A^{-1}\left[\frac{1}{v^{3}}\right]+a^{2} w_{0} A^{-1}\left[\frac{1}{v^{4}}\right] \\
=w_{0}+a w_{0} t+a^{2} w_{0} \frac{t^{2}}{2} .
\end{array}\right.
$$

In form general, we have

$w_{n}(t)=w_{0}+A^{-1}\left[\frac{1}{v} A\left[a w_{n-1}(t)\right]\right]=w_{0}\left(1+a t+\frac{a^{2} t^{2}}{2 !}+\ldots \ldots \ldots ..\right)$

for $n \rightarrow \infty, w_{n}(t)$ tends to the exact solution $w_{0} e^{a t}$.

Exemple2: Consider the following first oeder nonlinear logistic differential equation:

$\frac{d w(t)}{d t}=\alpha w(t)(1-w(t)), . . w(0)=\frac{1}{2}$

Using the above procedure and employing (9) leads to the iterative formula and the iteration formula , involving the Lagrange multiplier where $\lambda(v)=-\frac{1}{v}$ is given

$w_{n+1}(t)=w_{0}+A^{-1}\left[\frac{\alpha}{v} A\left[w_{n}\right]\right]-A^{-1}\left[\frac{\alpha}{v}\left[N\left(w_{n}\right)\right]\right] \ldots$

$w_{0}(t)=\frac{1}{2}$, is an initial approximation of Eq (16). Let $w_{n}=\sum_{i=0}^{n} u_{i}$, and nonlinear term can be decomposed as $N[w]=\sum_{i=0}^{\infty} B_{i}=F(u)$, where $B_{i}$ is the Adomian polynomials, then the Adomian series of the term $u^{2}$ reads $\left\{\begin{array}{l}B_{0}=F\left(u_{0}\right)=u_{0}^{2} \\ B_{1}=u_{1} F^{\prime}\left(u_{0}\right)=2 u_{1} u_{0} \\ B_{2}=u_{2} F^{\prime}\left(u_{0}\right)+\frac{1}{2} u_{1}^{2} F^{\prime \prime}\left(u_{0}\right)=2 u_{2} u_{0} \\ \vdots\end{array}\right.$
And
$\left\{\begin{array}{l}u_{n+1}=A^{-1}\left[\frac{\alpha}{v}\left[A\left(u_{n}\right)-A\left(B_{n}\right)\right]\right] \\ u_{0}=w_{0}=\frac{1}{2} .\end{array}\right.$ 
with symbolic computation, we can derive the following approximate solutions

$$
\begin{aligned}
& u_{1}=A^{-1}\left[\frac{\alpha}{v}\left[A\left(u_{0}\right)-A\left(B_{0}\right)\right]\right]= \\
& =A^{-1}\left[\frac{\alpha}{v}\left[\frac{1}{2 v^{2}}-\frac{1}{4 v^{2}}\right]\right]=A^{-1}\left[\frac{\alpha}{4 v^{3}}\right]=\frac{\alpha t}{4} \\
& \left\{u_{2}=A^{-1}\left[\frac{\alpha}{v}\left[A\left(u_{1}\right)-A\left(B_{1}\right)\right]\right]=A^{-1}\left[\frac{\alpha}{v}\left[\frac{\alpha}{4 v^{3}}-\frac{\alpha}{4 v^{3}}\right]\right]=0\right. \text { so the exact solution is given by } \\
& u_{3}=-\frac{\alpha^{3} t^{3}}{48} \\
& w(t)=\lim _{n \rightarrow \infty} w_{n}(t)=\lim _{n \rightarrow \infty}\left(\frac{1}{2}+\frac{\alpha t}{4}-\frac{\alpha^{3} t^{3}}{48}+\ldots \ldots \ldots .\right)=\frac{1}{1+e^{-\alpha t}}
\end{aligned}
$$

\subsection{Variable Coefficient Ordinary Equation}

Now, consider the following linear equation(4) with variable coefficients and assume the linear term as

$$
Q[w(t)]=a Q_{1}[w(t)]+b(t) Q_{2}[w(t)]
$$

where $Q_{1}, Q_{2}$ are also the linear operator, $a$ is a constant and $b(t)$ is a variable coefficient, then the new modified VIM is given as follows:

Takinging the Aboodh transform on both sides of eq(4) leads to the iterative formula

$$
\begin{aligned}
& W_{n+1}(v)=W_{n}(v)+\lambda(v)\left[v^{m} W_{n}(v)-\sum_{k=0}^{m-1} \frac{1}{v^{2-m+k}} w^{(k)}\left(0^{+}\right)\right] \\
& +A\left[a Q_{1}[w(t)]+b(t) Q_{2}[w(t)]\right]+A[P(w)]-A[g(t)] \ldots \ldots .
\end{aligned}
$$

Considering $A\left[a Q_{1}[w(t)]+b(t) Q_{2}[w(t)]\right]$ as restricted terms, the Lagrange multiplier will be easily obtain, then repeat the above iteration process.

Example 3. Consider the following differential equations with a variable coefficient $\frac{d w(t)}{d t}-(1+2 t) w(t)=0, \quad w(0)=1$

Applying the Aboodh transform on both sides of eq(21), we have

$v W(v)-\frac{1}{v}-W(v)-A[2 t w(t)]=0$

construct the variational iteration formula

$W_{n+1}=W_{n}+\lambda(v)\left[v W(v)-\frac{1}{v}-W(v)-A\left[2 t w_{n}(t)\right]\right]=0 \ldots$

Considering the term $A[2 t w(t)]$ is restricted variations, take the classical variation operator on both sides of eq(22) $\delta W_{n+1}=\delta W_{n}+\lambda(v)[(v-1)] \delta W_{n}(v) \ldots .(23)$

a Lagrange multiplier can be determined as $\lambda(v)=\frac{-1}{v-1}$.

Taking inverse Aboodh transform $A^{-1}$, the iteration formula eq (22) can be explicitly given as 


$$
\left\{\begin{array}{l}
w_{n+1}(t)=w_{n}(t)-A^{-1}\left[\frac{1}{v-1}\left[v W(v)-\frac{1}{v}-W(v)-A\left[2 t w_{n}(t)\right]\right]\right] \\
=w_{0}(t)+A^{-1}\left[\frac{1}{v-1}\left[A\left[2 t w_{n}(t)\right]\right]\right]
\end{array}\right.
$$

where $w_{0}(t) \mathrm{s}$ an initial approximation of eq (21). Then, we have

$$
w_{0}(t)=A^{-1}\left[\frac{-1}{v-1}\left(\frac{-1}{v}\right)\right]=A^{-1}\left[\frac{1}{v^{2}-v}\right]=e^{t} \text {, }
$$

after a series of computation, we can derive the following approximate solutions

$$
\left\{\begin{array}{l}
w_{1}(t)=w_{0}(t)+A^{-1}\left[\frac{1}{v-1}\left[A\left[2 t w_{0}(t)\right]\right]\right] \\
=e^{t}+A^{-1}\left[\frac{1}{v-1}\left[A\left[2 t e^{t}\right]\right]\right]=e^{t}+t^{2} e^{t}, \\
w_{2}(t)=w_{0}(t)+A^{-1}\left[\frac{1}{v-1}\left[A\left[2 t w_{1}(t)\right]\right]\right] \\
=e^{t}\left(1+t^{2}+\frac{t^{2}}{2}\right), \\
w_{3}(t)=w_{0}(t)+A^{-1}\left[\frac{1}{v-1}\left[A\left[2 t w_{2}(t)\right]\right]\right] \\
=e^{t}\left(1+t^{2}+\frac{t^{2}}{2}+\frac{t^{4}}{3 !}\right)
\end{array}\right.
$$

For $n \rightarrow \infty, w_{n}(t)$ tends to the exact solution $e^{t+t^{2}}$

\section{CONCLUSION}

In this paper, exact solutions of nonlinear differential equations are obtained using Aboodh transformation and differential transformation methods. This method is more efficient and easy to manage such differential equations. The results reveal that this method is very efficient, simple and can be applied to other nonlinear problems.

\section{REFERENCES}

J. H. He, "Variational iteration method-A kind of nonlinear analytical technique: some examples", International Journal of Non-Linear Mechanics, Vol. 34 No. 4, pp. 699-708,1999.

J. H. He, X.H. Wu, "Variational iteration method: new development and applications", Computers and Mathematics with Applications, Vol. 54 No.7-8, pp.881-894, 2007.

Y. Liu, W. Chen. A New Iterational Method for Ordinary Equations Using Sumudu Transform. Advances in Analysis, Vol. 1, No. 2, October 2016.

M. ELARBI BENATTIA, K. BELGHABA. Application of the Galerkin Method with Chebvshev
Polynomials for Solving the Integral Equation. Journal of Computer Science and Computational Mathematics, DOI: 10.20967/jcscm.2017.03.008.

M. ELARBI BENATTIA, K. BELGHABA. Numerical Solution for Solving Fractional Differential Equations using Shifted Chebyshev Wavelet. General Letters in Mathematics Vol.3, No.2, Oct 2017, pp.101-110.

G. C. Wu, "Challenge in the variational iteration method - A new approach to identification of the lagrange multipliers", Journal of King Saud University-Science, Vol. 25 No. 2, pp. 175178, 2013.

G. C. Wu, "Laplace transform overcoming principle draw backs in application of the variational iteration method to fractional heat equations", Thermal Science, Vol. 16 No. 4, pp. 1257-1261,2012.

Rahmatullah IBRAHIM NURUDDEEN. Aminu M. NASS, Exact Solutions of Wave-Type Equations By The Aboodh Decomposition Method. Stochastic Modeling and Applications. Vol.21, No. 1 (June, 2017), 23-30. 
Mohyud-Din, S. T., and Yildirim, A., 2010. Variational iteration method for delay differential equations using He's polynomials. Z. Naturforch, 65a, 1045-1048.

A. M. Wazwaz, "A comparison between the variational iteration method and Adomian decomposition method", Journal of Computational and Applied Mathematics, Vol. 207 No. 1, pp. 129-136, 2007.
A. M. Wazwaz, "The variational iteration method for analytic treatment for linear and nonlinear ODEs", Applied Mathematics and Computation, Vol. 212 No. 1, pp. 120-134, 2009.

M. A. Noor, "Some iterative methods for solving nonlinear equations using homotopy perturbation method", International Journal of Computer Mathematics", Vol. 87 No. 1, pp. 141- $\quad 149,2010$. 